\title{
A sighted aerial robot with fast gaze and heading stabilization
}

\author{
(Authors version) - doi:10.1109/IROS.2007.4399497 \\ Proceedings of the 2007 IEEE/RSJ International Conference on Intelligent Robots and Systems \\ San Diego, CA, USA, Oct 29 - Nov 2, 2007 \\ L. Kerhuel, S. Viollet, Member, IEEE, and N. Franceschini
}

\begin{abstract}
Autonomous guidance of Micro-Air Vehicles (MAVs) in unknown environments is a challenging task because these artificial creatures have small aeromechanical time constants, which make them prone to be disturbed by gusts of wind. Flying insects are subject to quite similar kinds of disturbances, yet they navigate swiftly and deftly. Flying insects display highperformance visuo-motor control systems that have stood the test of time. They can therefore teach us how vision can be used for immediate and vital actions.

We built a 50-gram tethered aerial demonstrator, called OSCAR II, which manages to keep its gaze steadily fixating a target (a dark edge), in spite of nasty thumps that we deliberately gave to its body with a custom-made "slapping machine". The robot's agile yaw reactions are based on:

- a mechanical decoupling of the eye from the body

- an active coupling of the robot's heading with its gaze

- a Visual Fixation Reflex (VFR)

- a Vestibulo-Ocular Reflex (VOR)

- an accurate and fast actuator (Voice Coil Motor, VCM)

The actuator is a 2.4-gram voice coil motor that is able to rotate the eye with a rise time as small as $12 \mathrm{~ms}$, that is, much shorter than the rise time of human oculo-motor saccades. In connection with a micro-rate gyro, this actuator endows the robot with a high performance "vestibulo ocular reflex" that keeps the gaze locked onto the target whatever perturbations in yaw affect the robot's body. Whenever the robot is destabilized (e.g., by a slap applied on one side), the gaze keeps fixating the target, while being the reference to which the robot's heading is servoed. It then takes the robot only $0.6 \mathrm{~s}$ to realign its heading with its gaze.
\end{abstract}

\section{ACRONYMS}

VFR Visual Fixation Reflex

VOR Vestibulo-Ocular Reflex

VCM Voice Coil Motor

FOV Field Of View

EMD Elementary Motion Detector

ZSL Zero-Setting Limiter

GCS Gaze Control System

HCS Heading Control System

\section{INTRODUCTION}

Ever since the first visual systems appeared in the Cambrian era, selection pressure led many living creatures to stabilize their line of sight (i.e., their gaze). Navigating in $3 \mathrm{D}$ environments, hovering [1], tracking mates [2] and intercepting prey [3] are among the behavioural feats that

L. Kerhuel, S. Viollet and N. Franceschini are with the Biorobotics Dept at the Movement and Perception Institute, CNRS/Univ de la Méditerranée CP 938, 163, avenue de Luminy, 13288 Marseille Cedex 09, France (email: lubin@kerhuel.eu, \{stephane.viollet, nicolas.franceschini \}@univmed.fr) flying insects achieve under visual guidance. Recent studies in free-flying flies have shown that these animals maintain their gaze fixed in space for about $100 \mathrm{~ms}$ episodes, using very fast reflexes [4]. In the vertebrates too, eye movements are the fastest and the most accurate movements generated.

Gaze stabilization is a difficult task to achieve by all animals because the eye actuator must be both :

- fast, to compensate for sudden, untoward disturbances.

- accurate, to ensure stable visual fixation.

In the flying fly, an active mechanism for gaze stabilization prevents the insect from being flummoxed adversely affected by disturbances affecting its flight such as vibrations or body jerks that may result from turbulences [4], [5]. Such a mechanism is far beyond what present-day robotics can achieve.

Several studies have considered implementing an active gaze stabilization system into mobile robots. A gaze control system combining a retinal position measurement with an inertial measurement has been proposed [6], and its performances were assessed qualitatively using slow perturbations applied by hand. Shibata and Schaal [7] described a gaze control system based on an inverse model of the mammalian oculomotor plant. Enhanced by a learning network, this system was able to decrease the retinal slip 4-fold for sinusoidal perturbations applied at moderate frequencies (up to $0.8 \mathrm{~Hz}$ ). Likewise, an adaptive image stabilizer for a robotic agent was built and tested by applying perturbations at moderate frequencies (up to $0.6 \mathrm{~Hz}$ ) [8]. Two other gaze stabilization systems inspired by the human Vestibulo-Ocular Reflex (VOR) have been presented [9], [10], but their performances have not been assessed quantitatively on a test-bed yet. Twombly [11] has made simulation experiments on a neuro-vestibular control system aimed at endowing a walking robot with active image stabilization. In the humanoid research field, a few robotic realizations have highlighted the need for stabilizing the gaze by using visuo/inertial oculomotor reflexes (e.g.: [8]). Wagner et al. built a fast responding oculomotor system [12] using air bearings and bulky galvanometers. None of the technological solutions ever proposed are compatible, however, with the stringent constraints imposed upon Micro-Air Vehicles (MAVs).

Gaze stabilization mechanisms in flying insects, particularly in flies, are a paragon of oculomotor reflexes that are key to heading stabilization. These high performance reflexes are of particular interest to the design of tomorrow's terrestrial, aerial, underwater and space autonomous vehicles with 
fast dynamics. Visually mediated stabilization of heading requires:

- a mechanical decoupling of the eye from the body (via a neck, as in flies, or via the orbit, as in the vertebrate visual system).

- an accurate and fast actuator for orienting the gaze. A fly has no less than 23 pairs of micro-muscles to control its gaze [13].

- a Visual Fixation Reflex (VFR) that keeps the gaze steadily on the target.

- a Vestibulo-Ocular Reflex (VOR), i.e., an active inertial reflex that rotates the eye in counter phase with the head. Flies typically exhibit such an inertial reflex, in particular for roll, based on the halteres gyroscopic organ [5]. A similar system was developed a hundred million years later in mammals, including humans. Rhesus monkeys' VOR react in the $0.5-5 \mathrm{~Hz}$ [14] and even $5-25 \mathrm{~Hz}$ [15] frequency ranges, thus exhibiting higher VOR performances than humans.

- an active coupling of the robot's heading with the gaze, via oculo-motor reflexes.

- a proprioceptive sensor able to measure the angular position of the eye in the head or in the body. Though still controversial in the case of the primate oculomotor system [16] this sensor exists in flies in terms of a pair of mechanosensory hair fields in the neck region [17], [18] that allow head-body angular deviations in pitch [4], roll [5] and yaw [19] to be measured and compensated for. In section 2, we describe our current aerial robot, called OSCAR II. OSCAR II differs from the original (OSCAR I [20], [21]) robot in that the eye is now mechanically uncoupled from the body, a configuration that will permit the gaze to be actively locked onto the target, whatever disturbances may affect the robot's body. In Section 3, we describe the scheme underlying the fast and accurate control of the "eye-in-robot" angle. In section 4, we explain how we merged a Gaze Control System (GCS) with a Heading Control System (HCS). In section 5, the robot's yaw control strategy is detailed, and we demonstrate the unusual performances attained for the overall gaze and heading control systems, which are both able to counteract nasty thumps given to the robot's body.

\section{DESCRIPTION OF THE OSCAR II ROBOT}

OSCAR II is a miniature (50-gram) twin-engine aerial platform equipped with a one-axis (horizontal) oculomotor mechanism (Fig. 1).

The seeing robot is able to adjust its heading accurately about the yaw axis by driving its two propellers differentially, via a custom-made dual sensorless speed governor (not described here). The robot's "body" consists of a carbon housing enclosing the two motors. It is prolonged on each side by a hollow beam within which the propeller carbon drive shaft can turn frictionlessly on micro ball bearings (Fig. 1). The robot's "head" is a large (diameter 15mm) carbon tube mounted firmly onto the motor housing. Within the head, an inner carbon "eye tube" mounted on pivot bearings

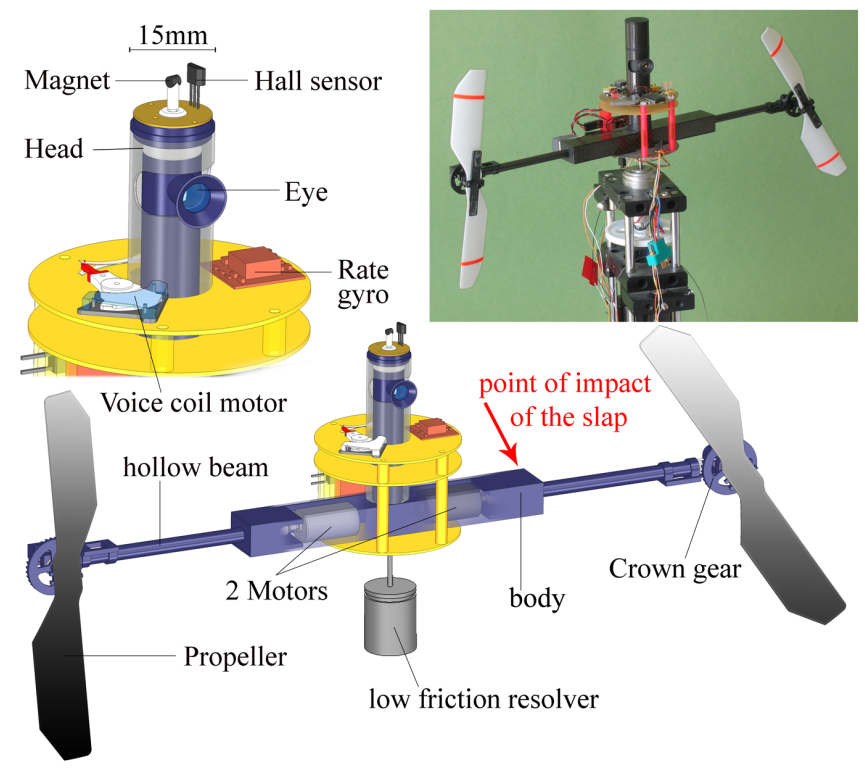

Fig. 1. OSCAR II is a 50-gram aerial robot that is able to control its heading about the vertical (yaw) axis by driving its two propellers differentially on the basis of what it sees. The eye of OSCAR II is mechanically uncoupled from the head, which is itself fixed to the "body". A Gaze Control System (GCS) (Fig. 5) allows the robot to fixate a target (a vertical white-dark edge placed 60 centimeters ahead), thus stabilizing its gaze despite severe disturbances (gusts of wind, slaps) that may affect its body. A Heading Control System (HCS) (Fig. 5), merged with the GCS, allows the robot's heading to catch up with the gaze, and thus to stabilize the heading in the same direction as the gaze. The right inset shows the current version of the OSCAR II robot mounted onto a low-friction, low-inertia resolver that allows its heading to be monitored accurately.

can turn freely about the yaw axis. The eye tube is springloaded between a pivot bearing (at the bottom part) and a micro-conical ball bearing (top part) through which a steel axle passes freely. A micromagnet glued to the tip of this axle allows the eye azimuthal orientation $\Theta_{e r}$ in the robot to be measured by a Hall sensor.

The robot's eye consists of a miniature lens (diameter $5 \mathrm{~mm}$, focal length $8.5 \mathrm{~mm}$ ) with an elementary "retina". The retina, composed of a single pair of matched PIN photodiodes, scans at $10 \mathrm{~Hz}$ by means of a fast piezo bender (Physik Instrumente) driven by an onboard waveform generator circuit (for details, see [22]). This retinal microscanning process is inspired by the one we identified in the fly compound eye [23]. The microscanning motion of the two photoreceptors occurs perpendicularly to the lens' axis, making their lineof-sights deviate in concert periodically. For details on the whys and wherefores of the particular microscanning law adopted, the reader is referred to our original analyses and simulations of the OSCAR sensor principle [24]. In essence, we showed that the association of an exponential scan with an Elementary Motion Detector (EMD) gives rise to a genuine Angular Position Sensor that is able to sense the position of an edge or a bar at high accuracy within its relatively small Field Of View (FOV) ( $F O V= \pm 1.4$ deg, i.e., about equal to the FOV of the human fovea). We showed that this sensor boasts a 40 -fold better angular resolution than the inter-receptor angle, in the task of locating an edge, and can 
therefore be said to be endowed with hyperacuity [25]. For further details on the performances (accuracy, calibration) of this microscanning visual sensor, the reader is referred to [22].

\section{IMPLEMENTATION OF THE ROBOT'S OCULOMOTOR SYSTEM}

In the human oculomotor system, extra-ocular muscles (EOM) show performances that are often deemed contradictory. On the one hand, they are required to maintain the gaze fixated accurately onto a steady target [26]. On the other hand, they are required to rotate the eye with a very small response time: a saccade of moderate amplitude takes only about $100 \mathrm{~ms}$ [27]. We emulated the high performance human oculomotor system by controlling the orientation of the eye-tube with an unconventional extra-ocular actuator: a Voice Coil Motor (VCM) that was dissected out from a hard disk microdrive (Hitachi). A VCM (normally used to displace the read/write head in disk drive control systems [28]) makes no trade-off between high positional accuracy and fast displacement.

Fig. 2 shows a top view scheme of the novel miniature oculomotor system that we built and installed on OSCAR II's body.

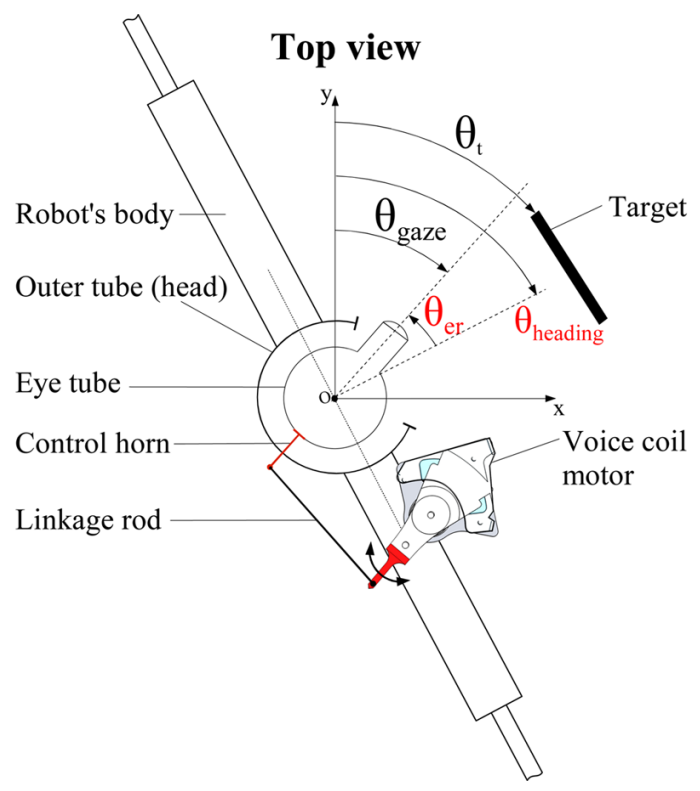

Fig. 2. OSCAR II oculomotor mechanism (top view). The central "eye tube" equipped with its two-pixel piezo-scanning retina (not shown here) is inserted into a larger carbon tube ("the head") that is mounted firmly onto the robot's body. The eye tube is mechanically uncoupled from the head with one degree of freedom about the yaw axis. The angle $\theta_{e r}$ between the robot's heading and the gaze is finely controlled (via the linkage rod and the control horn) by a micro VCM extracted from a hard disk microdrive. The visual sensor's output is a linear and even function of $\theta_{t}-\theta_{\text {gaze }}$.

VCM control requires an accurate position feedback loop. We used a simple PID structure to servo the angular position "eye in robot" $\theta_{e r}$ to the reference input $U_{e}$ (Fig. 3). $\theta_{e r}$ was measured by placing a Hall sensor in front of a micro magnet $\left(1 \mathrm{~mm}^{3}\right)$ glued to the eye-tube's rotation axle (see Fig. 1, left inset). All the transfer functions of Fig. 3 and 5 are detailed in the appendix.

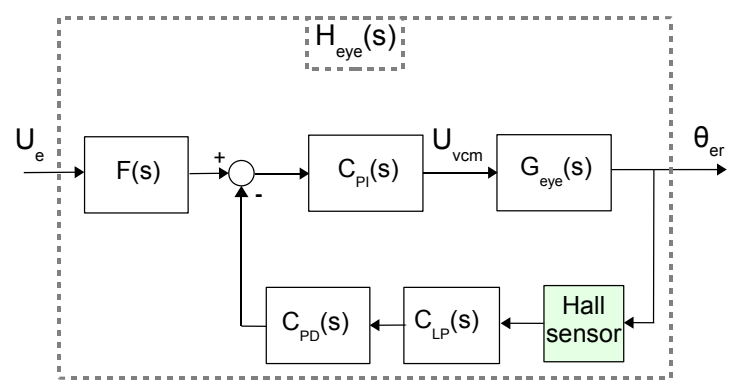

Fig. 3. Block diagram (noted $H_{\text {eye }}(s)$ ) of the Voice Coil Motor (VCM) servo system that makes the "eye in robot" angle $\theta_{e r}$ follow the reference input $U_{e}$. The $G_{\text {eye }}$ transfer function models the dynamic of the VCM loaded by the eye. $C_{P I}$ and $C_{P D}$ are the proportional-integral and proportional-derivative terms of the feedback controller, respectively. The $C_{L P}$ transfer function corresponds to a low pass filter that removes high frequency noise brought about by the Hall sensor. $F(s)$ is a $2^{\text {nd }}$ order low-pass filter that limits the step response overshoot. $F(s), C_{P I}, C_{L P}$ and $C_{P D}$ are digitized (sample rate $1 \mathrm{~ms})$ and implemented in the same dsPIC microcontroller.

The output of the Hall sensor is noisy. Though the high frequency noise (above $200 \mathrm{~Hz}$ ) has little impact on the VCM position, it markedly increases the electrical consumption of the VCM. We therefore used a low pass filter $\left(C_{L P}\right)$, with a cut-off frequency of $200 \mathrm{~Hz}$. This filter does not affect the overall performances of the position feedback loop.

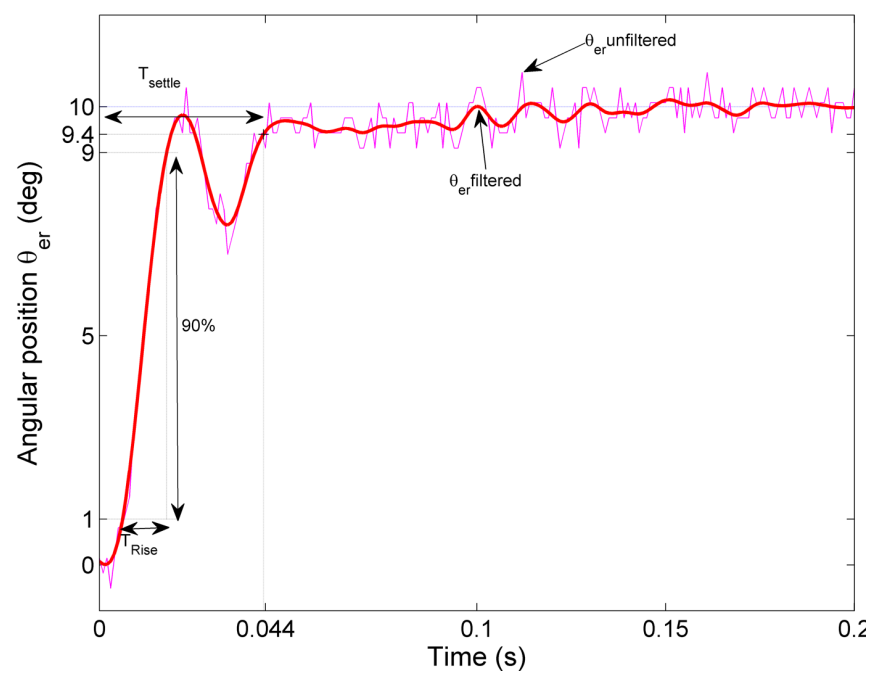

Fig. 4. Response of the "eye in robot" angular position $\theta_{e r}$ to a large (10-degree) step input applied to the reference input $U_{e}$ (Fig. 3). To improve the readability, the red curve is the filtered version of the raw $\theta_{e r}$ data (cyan curve) using a $4^{\text {th }}$ zero phase delay filter (cut-off frequency at $40 \mathrm{~Hz}$ ). The use of a voice coil motor actuator allows the closed loop rise time to reach an unusually small value $\left(T_{\text {rise }}=12 \mathrm{~ms}\right)$.

The step response shown in Fig. 4 illustrates the very fast dynamics attained in controlling the orientation of the eye in the robot: $\theta_{\text {er }}$ has a settling time $T_{\text {settle }}$ (at 94\%) as small as $44 m s$ and a rise time $T_{\text {rise }}$ as small as $12 \mathrm{~ms}$. The mean velocity attained during the rising phase is $660 \mathrm{deg} / \mathrm{s}$, which is higher than the velocity $(180 \mathrm{deg} / s)$ reached in our 


\section{Heading control system (HCS)}

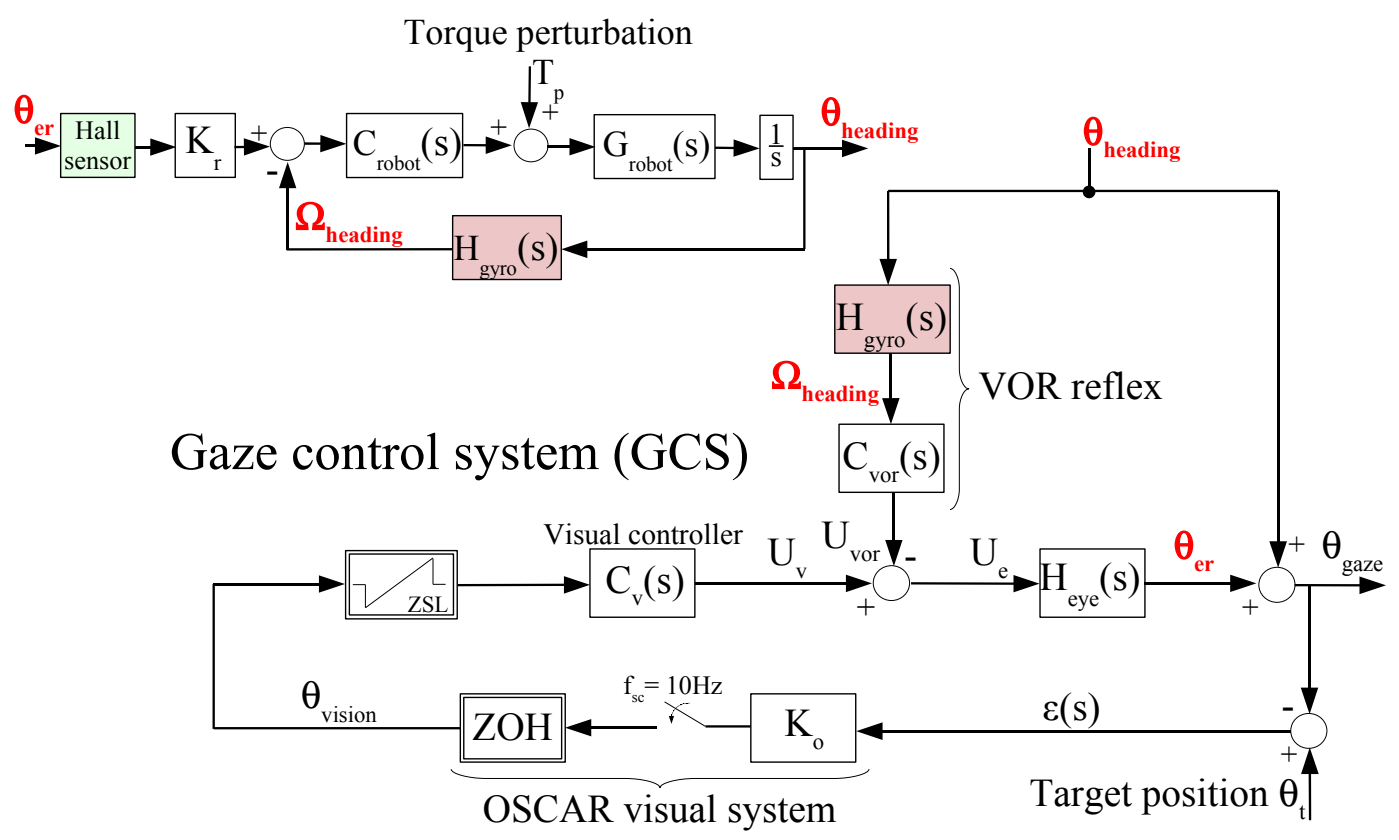

Fig. 5. Block diagrams of the two intertwined control systems (an HCS and a GCS) which are implemented onboard the OSCAR II robot. The GCS keeps the gaze $\left(\theta_{\text {gaze }}\right)$ locked onto a stationary target (bearing $\left.\theta_{t}\right)$, despite heading disturbances $\left(T_{p}\right)$. This system is composed of a visual feedback loop based on the OSCAR visual sensor (which acts as an "angular position sensor device") and a feedforward control system emulating the Vestibulo-Ocular-Reflex (VOR). The HCS makes $\theta_{\text {heading }}$ servoed to $\theta_{e r}$ by acting differentially onto the rotational speed of each propeller. Since $\theta_{\text {heading }}$ is also an input disturbance to the GCS, any changes in the heading (due to a torque perturbation applied to the robot) is compensated for by a counter-rotation of the eye ( $\theta_{e r}$ angle). A null value of $\theta_{e r}$ means that $\theta_{\text {heading }}=\theta_{\text {gaze }}$. Note that the two proprioceptive signals $\theta_{e r}$ and $\Omega_{\text {heading }}$, given by the Hall effect sensor and the rate gyro (cf. Fig. 1), respectively, are used in both the GCS and the HCS.

previous design [22] and even higher than the saturation velocity of the human eye $(500 \mathrm{deg} / \mathrm{s})$ measured during a saccade [27]. Unlike our robot's oculomotor control system (which is essentially linear), the human oculomotor control system is nonlinear, however, with a rise time that typically grows with the saccade amplitude [27].

\section{A gAZE CONTROL SYSTEM THAT DRIVES A HEADING CONTROL SYSTEM}

\section{A. The Gaze Control System (GCS)}

Fig. 5 shows that the control signal $U_{e}$ of the eye results from the simple subtraction of two control signals:

- $U_{v}$, an angular position signal arising from the visual feedback controller.

- $U_{V O R}$, an angular position signal arising from the inertial (feedforward) controller

A VOR feedforward control pathway was implemented, which acts like its biological counterpart. Like the semi circular canals of the inner ear, which give an estimation of the head angular speeds [29], a Micro-Electro-Mechanical System (MEMS) rate gyro (Analog Devices ADXRS300) measures the robot's body yaw velocity. The VOR reflex aims at making any change in $\theta_{e r}\left(\Delta \theta_{e r}\right)$ follow a change in $\theta_{\text {heading }}\left(\Delta \theta_{\text {heading }}\right)$ faithfully but with opposite sign. In the frequency domain, this will occur only if the gain and phase of the transfer function relating $\theta_{e r}$ to $\theta_{\text {heading }}$ are held at $0 \mathrm{~dB}$ and $0 \mathrm{deg}$, respectively, over the largest possible frequency range. This leads to a theoretical expression for $C_{V O R}$ as follows:

$$
C_{V O R_{t h}}(s)=H_{\text {gyro }}^{-1}(s) H_{\text {eye }}^{-1}(s)
$$

Stability problems caused by the high static gain introduced by the pseudo integrator $H_{\text {gyro }}^{-1}(s)$ led us to adopt an approximation noted $\hat{H}_{g y r o}^{-1}(s)$. The expression of $C_{V O R}$ therefore becomes:

$$
C_{V O R}(s)=\hat{H}_{\text {gyro }}^{-1}(s) H_{\text {eye }}^{-1}(s)
$$

Therefore, if the robot's heading is subjected to a rotational disturbance, the change in $\theta_{\text {heading }}$ will be measured and compensated for by the VOR feedforward control system that will impose a counter rotation of the eye of similar amplitude.

It can be seen in Fig. 5 that $\theta_{\text {heading }}$ also acts in a parallel way as an input disturbance to the visual feedback. The control signal $U_{v}$ derived from the visual controller $C_{v}(s)$ acts upon the orientation $\theta_{e r}$ of the eye so as to compensate for this disturbance, thus holding the gaze $\theta_{\text {gaze }}$ effectively in the direction $\theta_{t}$ of the visual target (that is, making $\varepsilon=0$ in Fig. 5, bottom right).

We showed that $\Delta \theta_{e r}$ follows $\Delta \theta_{\text {heading }}$ faithfully but only over a limited frequency range (between $1 \mathrm{~Hz}$ and $11 \mathrm{~Hz}$, 
data not shown here). This limitation is due to both the modification made on $C_{V O R}$ (for stability consideration) and the approximations made during the identification of the transfer functions $H_{\text {gyro }}(s)$ and $H_{\text {eye }}(s)$.

As described in the appendix, the visual controller $C_{v}(s)$ (see Fig. 5) is an integrator. This means that the visual controller copes with any target displacement without introducing any steady state error $\left(\varepsilon=\theta_{t}-\theta_{\text {gaze }}\right.$ in Fig. 5). In other words, there is no "retinal slip error" in the steady state. To prevent runaway of the eye when it loses sight of a target, we developed a special limiter [21], which we have called a Zero-Setting Limiter (ZSL), and introduced it upstream from the visual controller (Fig. 5). The purpose of this nonlinear block is to clamp the error signal back to zero whenever the target gets out of the FOV.

Due to its scanning frequency of $10 \mathrm{~Hz}$ [24], the OSCAR II visual sensor inevitably introduces a latency of $100 \mathrm{~ms}$ into the visual feedback loop. This latency is the main limiting factor in the process of visually rejecting the (fast) disturbances that may affect the robot. The VOR reflex acts in a complementary manner, improving the dynamics of gaze stabilization dramatically, and thus preventing the fixated target from being led astray outside the (narrow) FOV of the eye.

\section{B. The Heading Control System (HCS)}

A major novelty of the present work is the merging of the visuo-inertial reflex described above with the heading control system of the OSCAR II robot. We designed an HCS that takes into account the yaw dynamics of the robot, modeled by the transfer function $G_{\text {robot }}(s)$. The HCS involves

- a measurement of the robot's yaw angular speed $\Omega_{\text {heading }}$ (yielded by the same rate gyro as used in the VOR)

- a proportional-integral controller (included in $C_{\text {robot }}(s)$ )

In the steady state, the angle $\theta_{e r}$ is null (see Fig.3), which means that the HCS acts so as to make $\theta_{\text {heading }}$ equal to $\theta_{\text {gaze }}$ (zero steady-state error).

The implementation of the HCS (top part of Fig. 5) means that the robot's orientation $\left(\theta_{\text {heading }}\right)$ is controlled through the orientation of the eye in the robot, $\theta_{e r}$. These two angles are therefore actively coupled. The fact that the robot "carries the eye" means that $\theta_{\text {heading }}$ is both an input disturbance for the VFR based on the OSCAR visual system and an input signal to the rate gyro that serves both to the VOR reflex and to the velocity feedback loop of the HCS.

To summarize, both the GCS and the HCS are intertwined and share the same two proprioceptive sensors: (i) the Hall effect sensor that delivers a signal proportional to $\theta_{\text {er }}$ (green in Fig.3 and 5) and (ii) the rate gyro that delivers $\Omega_{\text {heading }}$ (pink in Fig.5). Even though the GCS and the HCS loops are intimately nested, the GCS does not involve the robot's dynamics. This means that the two controllers present in the GCS can be tuned by taking into account only the dynamics of the disturbance $\theta_{\text {heading }}$ that needs to be rejected. This simplifies greatly the design of the overall control system.
The overall gaze and heading control system does not require large computational resources. The two digital controllers (the one for the the propeller speed control system at $70 \mu s$ sample rate -not described here- and the other one for the VCM based feedback control system at $1 \mathrm{~ms}$ sample rate) were implemented using a custom-made rapid prototyping tool for simulink : Embedded Target for Microchip dsPIC. The controllers involved in HCS and GCS (including the VOR and the visual feedback-loop) were digitized at $1 \mathrm{~ms}$ sample rate, by using Tustin method and implemented in the dSpace environment.

\section{HIGH PERFORMANCE GAZE AND HEADING STABILIZATION SYSTEM}

We characterized our miniature gaze and heading control system by applying drastic torque perturbations to the robot's body. We built a "slapping machine" based on a DC motor rotating at a constant speed, and a light wooden arm that was suddenly coupled to the motor drive via a miniature electromagnetic clutch. The slapping machine was placed so as to allow the wooden arm to hit the robot at the impact point indicated by the red arrow in Fig. 1. Brisk thumps were thus given to the robot every 5 seconds while its gaze was fixating a contrasting edge placed at a distance of $60 \mathrm{~cm}$ from the eye.

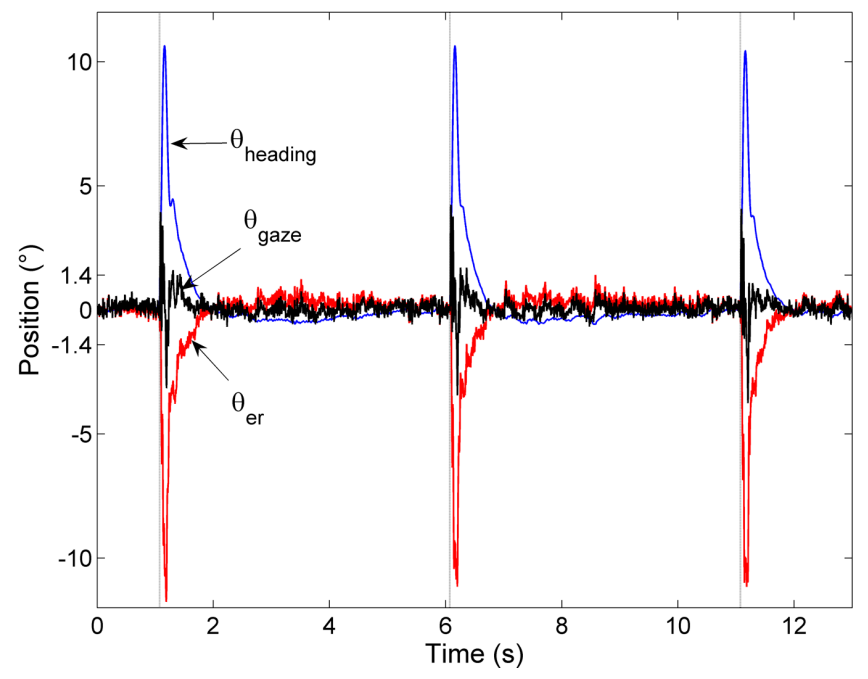

Fig. 6. Reaction of the robot $\left(\theta_{\text {heading }}\right)$, the "eye-in-robot" $\left(\theta_{e r}\right)$ and the gaze $\left(\theta_{\text {gaze }}\right)$ to a sequence of 3 thumps given to the robot every 5 seconds (the thin vertical lines locate the timing of each thump. These repetitive slaps were applied to the robot's body unilaterally (see the impact point of the slap in Fig. 1), using a wooden stick driven by a DC motor upon activation of an electromagnetic clutch. The sudden yaw perturbation is seen to be counteracted extremly rapidly (within $20 \mathrm{~ms}$ ) by the VOR reflex, which maintains the robot's gaze $\left(\theta_{\text {gaze }}\right)$ close to the target position $\theta_{t}$ (Here, $\theta_{t}$ is assumed to be 0$)$. The robot is then seen to reorient itself more rapidly (in about 0.6 second).

As can be seen from the HCS block diagram (Fig. 5, top), any torque perturbation $T_{p}$ will be compensated for by the controller $C_{\text {robot }}$. Meanwhile, however, the torque perturbation will have led inevitably to a transient change in heading. Since $\theta_{\text {heading }}$ acts as an input disturbance to the 


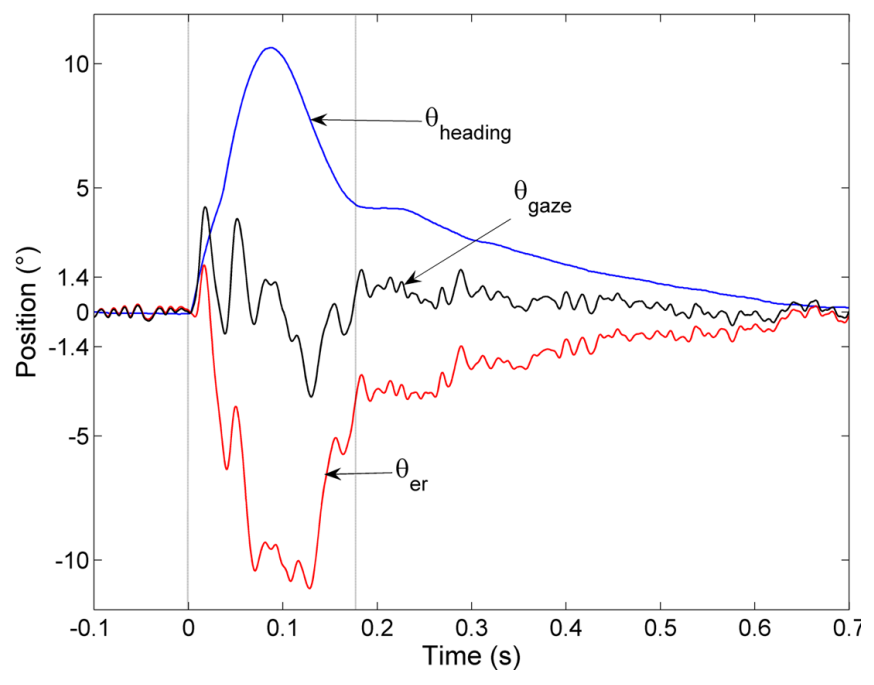

Fig. 7. Magnified version of the second thump given to the robot in Fig. 6 , showing how the VOR reflex compensates for the robot transient rotation caused by the nasty thump. The time at which the thump is given is shown by the left vertical line. The "eye-in-robot" profile $\left(\theta_{e r}\right.$ red curve) shows that the eye rotation immediately counteracts the robot rotation ( $\theta_{\text {heading }}$, blue curve), so that the gaze ( $\theta_{\text {gaze }}$, black curve) remains quasi-steady. The fast return phase (between $0 \mathrm{~ms}$ and $177 \mathrm{~ms}$ ) of the robot's heading is mainly due to the yaw velocity feedback loop of the HCS combined with the action of the VOR in the GCS. The $\theta_{\text {heading }}$ slow return phase (between $177 \mathrm{~ms}$ and $650 \mathrm{~ms}$ ) results from the control input signal $\theta_{e r}$ (Fig. 6). The VOR reflex operates quasi-instantaneously compared with the relatively long $(100 \mathrm{~ms})$ refresh period of the visual system. The left vertical line indicates the time at which the thump is given.

GCS, any torque perturbation is also compensated for by a counterrotation of the "eye-in-robot" $\theta_{e r}$. As a consequence, the robot re-orients its heading automatically with its gaze, until $\theta_{e r}$ becomes null again.

The robot was mounted onto the shaft of a low friction and low inertia resolver, which enabled its azimuthal orientation $\theta_{\text {heading }}$ to be monitored.

In all the experiments presented here, the absolute target angle $\theta_{t}$ is taken to be $\theta_{t}=0$ (see Fig. 2) for the sake of simplicity. All $\theta_{e r}$ response curves were filtred with a 0 phase delay 4th order low pass filter (cut-off frequency at $40 \mathrm{~Hz}$, see Fig.4) to remove the instrumentation noise.

Fig. 6 shows that $\theta_{\text {heading }}$ is violently (and reproducibly) perturbed by three sudden thumps. The eye can be seen to immediately counterrotate in the robot's body (see the curve $\theta_{e r}$ ), holding the gaze virtually locked onto the target (see the curve $\theta_{\text {gaze }}$ ).

Fig. 7 shows a close up of the robot's, eye's and gaze's responses to the second thump given in Fig. 6. Time 0 s corresponds here precisely to the time when the thump is given, as determined with a micro-accelerometer mounted at the tip of the inter-propeller beam. The "robot" response can be decomposed into two phases:

- A fast phase (between 0ms and 177ms) where the perturbation is rejected mostly by the velocity feedback loop of the HCS and by the VOR via the input signal $\theta_{\text {er }}$ (Fig. 5).

- A slow phase (between $177 \mathrm{~ms}$ and $650 \mathrm{~ms}$ ) where the

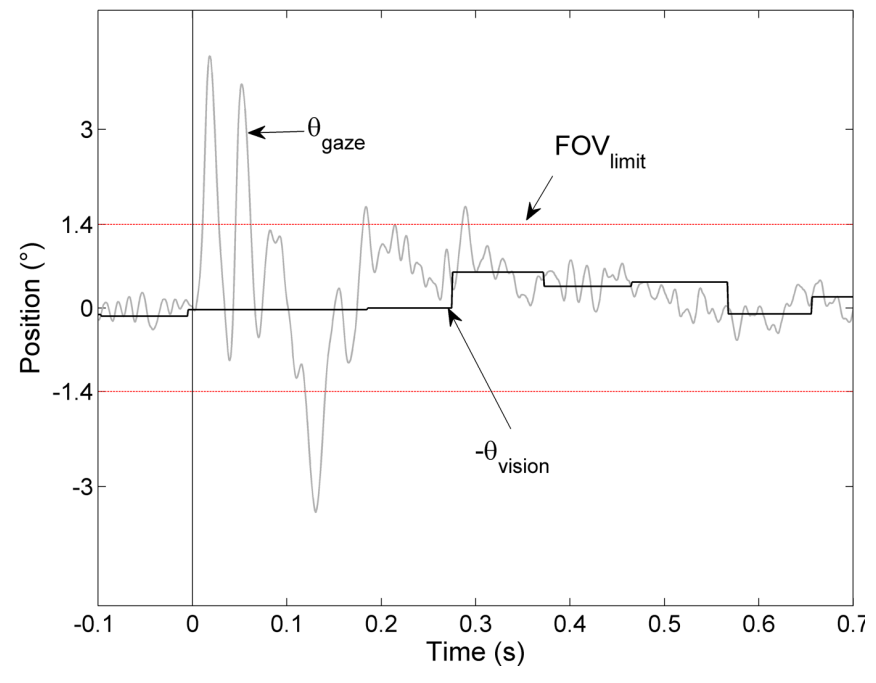

Fig. 8. Gaze orientation $\left(\theta_{\text {gaze }}\right)$ compared with the gaze orientation relative to the target $\left(\theta_{\text {vision }}\right)$ during the second second thump given to the robot in Fig. 6. The two horizontal red lines delimit the field of view FOV limit $( \pm 1.4 \mathrm{deg})$ of the eye. A value of the gaze higher than $\pm 1.4 \mathrm{deg}$ means that the target has wandered out of the field of view. The duration of this "lost target" phase is so short $(50 \mathrm{~ms}$, i.e. twice shorter than the vision refresh period) that it does not impair gaze stabilization.

perturbation is entirely rejected by both the VOR and the visual feedback loop.

As shown in Fig. 7, the eye position $\theta_{e r}$ counteracts the robot's position $\theta_{\text {heading }}$ quasi immediately thanks to the high speed dynamics of the eye's orientation feedback control system GCS based on the fast VCM actuator. The eye's rotation is fast enough to hold the gaze $\theta_{\text {gaze }}$ locked onto the target. Measurement of the robot's gaze $\left(\theta_{\text {gaze }}\right)$ was not directly accessible (it would have required, e.g., a magnetic search coil or an eye tracker). The gaze was therefore calculated according to its definition (see Fig. 2)

$$
\theta_{\text {gaze }}=\theta_{\text {heading }}+\theta_{\text {er }}
$$

Figure 8 shows that the thumps given to the robot's body were so severe that the contrasting target (a white-dark edge) transiently wandered out of the small, $\pm 1.4 \mathrm{deg}$ field of view of the eye. Yet the robot does not get flummoxed by this situation. The contrasting target keeps being "seen" by the eye (see the $\theta_{\text {vision }}$ signal), because the time during which the target is led astray of the visual field $(50 \mathrm{~ms})$ is shorter than the visual scanning refresh period of the eye $(100 \mathrm{~ms})$.

\section{CONCLUSION}

We described the principle and implementation of a miniature, tethered aerial platform equipped with a one-axis, ultrafast and accurate gaze and heading control system inspired by highly proficient, long existing biological systems. The role played by the overall control system is to hold the lineof-sight of the eye (i.e., the gaze) stabilized onto a sharp contrast edge - as do flies between two saccades [4] - in spite of the disturbances that may affect the body (carrying the eye). This is achieved via two processes, a GCS and a 
heading control system HCS that are largely interactive (Fig. 5). The GCS itself merges two reflexes:

- a slow but accurate Visual Fixation Reflex (VFR) that copes with long-lived (i.e., "DC") fixation

- a fast Vestibulo-Ocular Reflex (VOR) that copes with transient robot's angular perturbations.

The HCS reorients the robot's heading in the same direction as the gaze, a process that takes a relatively long time $(0.6$ seconds), due to the relatively large body inertia (Fig. 7).

We paid particular attention to testing the robustness of the control system by recording the eye's and robot's reactions to severe torque perturbations applied to the body. Using a custom-made "slapping machine", we showed that once destabilized by a nasty thump given to its body, the robot:

- keeps its gaze fixating the contrast edge, despite the small visual field of the eye

- reorients its heading actively until its catches up with the gaze direction (Figs. 6, 7, 8)

A highlight of this study is that the gaze itself is the fundamental (Eulerian) reference, about which any motor action (orienting the "eye in robot" and the "robot in space") is based.

This study considerably extends the scope of a former study in which we had realized a gaze control system endowed with a VOR, without showing its use onboard any robotic platform [22]. Besides, we now introduced a novel oculomotor servomechanism based on a VCM and a Hall sensor. The dynamics of this position servo is so high that it is able to bring the gaze to a new position in $12 \mathrm{~ms}$, i.e., much faster than a saccade in the human eye (Fig. 4).

The two control systems (Heading Control System (HCS) and Gaze Control System (GCS)) that we presented here are largely intertwined and interact strongly. We have shown that the HCS is actively coupled with the GCS by receiving as inputs $\theta_{e r}$ (as measured by the Hall sensor) and $\Omega_{\text {heading }}$ (as measured by the rate gyro). Even though the eye is mechanically uncoupled from the robot's body, the GCS is passively coupled to the HCS due to the fact that the robot "carries" the whole oculomotor system (which means that the heading can disturbs the gaze). The active and passive couplings between the two control systems make the high performances of the robot's heading control system (HCS) directly related to the high performances of the gaze control system (GCS). In other words, the faster the gaze stabilizes, the faster the heading will stabilize ; the more accurate the gaze, the more accurate the heading.

Our lightweight and robust gaze control system can be useful for the guidance of manned and Unmanned Air Vehicule (UAV), of Autonomous Underwater Vehicle (AUV), and particularly for Micro-Air Vehicle (MAV) and MicroUnderwater Vehicle (MUV), which are particularly prone to disturbances that may originate from fast pitch variations, wing-beats (or body undulations or fin-beats), wind gusts (or water streams), ground effects, Vortices, and all kinds of unpredictable aerodynamic (or hydrodynamic) disturbances.
Biological systems teach us that such disturbances are better compensated for early on by implementing a visuo-inertial gaze stabilization system that will yield a reference for heading stabilization. Anchoring the gaze on a feature of the environment provides a drift-free reference with respect to the world.

\section{ACKNOWLEDGEMENTS}

The authors acknowledge the assistance of M. Boyron for the design of the miniature electronic boards including the piezo driver, the EMD and the control systems. We thank F. Paganucci and Y. Luparini for their help with the mechanical construction of the eye, F. Ruffier and J. Serres for fruitful discussions and comments on the manuscript. This work was supported by CNRS, University of the Mediterranean and the French National Agency for Research (ANR, RETINAE project).

\begin{tabular}{|c|c|}
\hline \multicolumn{2}{|c|}{ APPENDIX } \\
\hline$H_{g y r o}(s)=K_{g} \frac{\left(\tau_{2} s+1\right)}{\left(\tau_{1} s+1\right)}$ & $\begin{array}{l}\text { With } \tau_{1}=4.3 * 10^{-3} \mathrm{~s}, \tau_{2}=1897.5 \mathrm{~s} \\
\text { and } K_{g}=2.27 * 10^{-6}\end{array}$ \\
\hline$\hat{H}_{\text {gyro }}^{-1}(s)=K_{\text {ginv }} \frac{\left(\tau_{5} s+1\right)}{\left(\tau_{6} s+1\right)}$ & $\begin{aligned} \text { With } \tau_{5} & =3.68 * 10^{-3} \mathrm{~s}, \tau_{6}=2.31 \mathrm{~s} \\
& \text { and } K_{\text {ginv }}=606.5\end{aligned}$ \\
\hline$H_{\text {eye }}(s)=K_{e} \frac{\left(\tau_{4} s+1\right)}{\left(\tau_{3} s+1\right)}$ & $\begin{array}{c}\text { With } \tau_{3}=18.7 * 10^{-3} \mathrm{~s}, \tau_{4}=0.5 * 10^{-3} \mathrm{~s} \\
\text { and } K e=226.3 * 0.9\end{array}$ \\
\hline$G_{\text {robot }}(s)=\frac{K_{r o b}}{\frac{1}{W_{r}^{2}} s^{2}+\frac{2 \zeta_{r}}{W_{r}} s+1}$ & $\begin{array}{c}\text { With } W_{r}=17.96 \mathrm{rads}^{-1}, \zeta_{r}=0.595 \\
\text { and } K_{r o b}=3.74\end{array}$ \\
\hline$K_{r}=6$ & Pure gain \\
\hline$C_{\text {robot }}(s)=3.7 * 10^{-6}$ & Pure gain \\
\hline$C_{V O R}(s)=\hat{H}_{\text {gyro }}^{-1}(s) H_{\text {eye }}^{-1}(s)$ & \\
\hline$C_{v}(s)=\frac{K_{0}}{s}$ & $\begin{array}{l}\text { With visual sampling rate } T_{s c}=0.1 \mathrm{~s} \\
\text { and } K_{0}=0.0574\end{array}$ \\
\hline$G_{\text {eye }}(s)=\frac{\left(\tau_{7} s+1\right)}{\left.\tau_{8}^{2} s^{2}+2 \tau_{8} \zeta_{\text {eye }} s+1\right)}$ & $\begin{array}{c}\text { With } \tau_{7}=484 * 10^{-6} s, \tau_{8}=26 * 10^{-3} \mathrm{~s} \\
\text { and } \zeta_{\text {eye }}=0.27\end{array}$ \\
\hline$C_{P D}(s)=K_{P D} \frac{\left(\tau_{9} s+1\right)}{\left(\tau_{10} s+1\right)}$ & $\begin{array}{c}\text { With } \tau_{9}=9.65 * 10^{-3} \mathrm{~s}, \tau_{10}=1.65 * 10^{-3} \\
\text { and } K_{P D}=0.8\end{array}$ \\
\hline$C_{L P}(s)=\frac{1}{\left(\tau_{L P} s+1\right)}$ & With $\tau_{L P}=769 * 10^{-6} \mathrm{~s}$ \\
\hline$C_{P I}(s)=K_{P I} \frac{\left(\tau_{P I} s+1\right)}{\tau_{P I} s}$ & With $\tau_{P I}=0.015 \mathrm{~s}$ and $K_{P I}=2.2$ \\
\hline$F_{s}(s)=\frac{1}{\frac{1}{W_{n}^{2}} s^{2}+\frac{2 \zeta_{n}}{W_{n}} s+1}$ & With $W_{n}=200 \mathrm{rads}^{-1}$ and $\zeta_{n}=0.9$ \\
\hline
\end{tabular}

\section{REFERENCES}

[1] R.Kern and D.Varjù, "Visual position stabilization in the hummingbird hawk moth, macroglossum stellatarum 1." J Comp Physiol A 182, pp. 225-237, 1998. 
[2] N.Boeddeker, R.Kern, and M. Egelhaaf, "Chasing a dummy target: smooth pursuit and velocity control in male blowflies," in Proc. $R$. Soc. Lond.B 270, 2003, pp. 393-399.

[3] R.M.Olberg, A. Worthington, and K.R.Venator, "Prey pursuit and interception in dragonfly," J Comp Physiol A186, pp. 155-162, 2000.

[4] J. H. V. Hateren and C. Schilstra, "Blowfly flight and optic flow. ii. head movements during flight," J. Exp Biol, vol. 202, pp. 1491-1500, 1999.

[5] R. Hengstenberg, "Mechanosensory control of compensatory head roll during flight in the blowfly calliphora erythrocephala meig," J. Comp Physiol A, vol. 163, pp. 151-165, 1988.

[6] T. Yamaguchi and H. Yamasaki, "Velocity based vestibular-visual integration in active sensing system," in Proc. IEEE Intern. Conf. on Multisensor Fusion and Integration for Intelligent System, Las Vegas, USA, 1994, pp. 639-646.

[7] T. Shibata and S. Schaal, "Biomimetic gaze stabilization based on feedback-error learning with nonparametric regression networks," $\mathrm{Neu}$ ral Networks, vol. 14, pp. 201-216, 2001.

[8] F. Panerai, G. Metta, and G. Sandini, "Learning visual stabilization reflexes in robots with moving eyes," Neurocomputing, vol. 48, pp. 323-337, 2002.

[9] A. Lewis, "Visual navigation in a robot using zig-zag behavior," in Proceedings of Neural Informations Processing Systems (NIPS), 1997, pp. 822-828.

[10] P. Viola, "Neurally inspired plasticity in oculomotor processes," in Proceedings of Neural Informations Processing Systems (NIPS), 1989, pp. 290-297.

[11] X.Twombly, R.Boyle, and S.Colombano, Active Stabilization of Images Acquired on a Walking Robotic Platform. G.Bebis et al. (Eds.), ISVC 2006, LNCS 4292, 2006, pp. 851-860.

[12] R. Wagner, I. W. Hunter, and H. L. Galiana, "A fast robotic eye/head system: Eye design and performance," in Proc. of IEEE Engineering in Medicine and Biology Society, vol. 14, 1992, pp. 1584-1585.

[13] N. J. Strausfeld, H. S. Seyan, and J. J. Milde, "The neck motor system of the fly calliphora erythrocephala. 1. muscles and motor neurons," J. Comp. Physiol, vol. A 160, pp. 205-224, 1987.

[14] E. L. Keller, "Gain of the vestibulo-ocular reflex in monkey at high rotational frequencies," Vis. Res., vol. 18, pp. 311-115, 1978.

[15] M. Huterer and K. E. Cullen, "Vestibuloocular reflex dynamics during high-frequency and high acceleration rotations of the head on body in rhesus monkey," J Neurophysiol, vol. 88, pp. 13-28, 2002.

[16] R. W. Clifford, P. C. Know, and G. N. Dutton, "Does extraocular muscle proprioception influence oculomotor control ?" Br. J. Ophtalmol, vol. 84 , pp. $1071-1074,2000$
[17] T.Preuss and R.Hengstenberg, "Structure and kinematics of the prosternal organs and their influence on head position in the blowfly calliphora erythrocephala meig," J Comp Physiol A 171, pp. 483-493, 1992.

[18] A. Paulk and C. Gilbert, "Proprioceptive encoding of head position in the black soldier fly, hermetia illucens (1.) (stratiomyidae)," The Journal of Experimental Biology, vol. 209, pp. 3913-3924, 2006.

[19] E. Liske, "The influence of head position on the flight behaviour of the fly, calliphora erythrocephala," J. Insect Physiol, vol. 23, pp. 375-179, 1977.

[20] S. Viollet and N. Franceschini, "Visual servo system based on a biologically-inspired scanning sensor," in Sensor fusion and decentralized control in robotics II, SPIE, vol. 3839, Boston, 1999, pp. 144-155.

[21] — Super-accurate visual control of an aerial minirobot, in: Autonomous Minirobots for Research and Edutainment AMIRE. Padderborn, Germany: U. Ruckert, J. Sitte and U. Witkowski (Eds), 2001.

[22] _ "A high speed gaze control system based on the vestibulo-ocular reflex," Robotic and Autonomous System, vol. 50, pp. 147-161, 2005.

[23] N. Franceschini and R. Chagneux, "Repetitive scanning in the fly compound eye," in Gottingen Neurobiol. Conf., Gottingen, 1997, p. 279.

[24] S. Viollet and N. Franceschini, "Biologically-inspired visual scanning sensor for stabilization and tracking," in Proceedings of IEEE IROS'99, Kyongju, Korea, 1999, pp. 204-209.

[25] G. Westheimer, Visual hyperacuity. Berlin: Ottoson, Sensory Physiology 1, Springer, 1981.

[26] R. M. Steinman, "Voluntary control of microsaccades during maintained monocular fixation," Sciences, vol. 155, pp. 1577-1579, 1967.

[27] W. Becker, Vision and visual dysfunction (Vol 8). GR.H.S. Carpenter (Ed) Macmillan Press, Ltd, 1991, ch. 5 : Saccades, pp. 95-137.

[28] B. M. Chen, T. H. Lee, K. Peng, and V. Venkataramanan, Hard Disk Drive Servo Systems 2nd Edition. Springer, Berlin, 2006.

[29] R. H. S. Carpenter, Movements of the eyes, 2nd edt. PION, London, 1988 , ch. 2 : Vestibular eye movements.

[30] F. Panerai, G. Metta, and G. Sandini, "Learning vor-like stabilization reflexes in robots," in ESANN'2000 Proceedings, Bruges,Belgium, April 2000, pp. 95-102.

[31] N. Strausfeld, Atlas of an Insect Brain. Springer-Verlag, Berlin, Heidelberg, 1976.

[32] S.Viollet, L. Kerhuel, and N. Franceschini, "A 1-gram dual sensorless speed governor for micro-air vehicles," in proc. IEEE IROS'07, San Diego, United States, submitted.

[33] S. Viollet and N. Franceschini, "A miniature biomimetic gaze control system," in proc. IEEE Int Conf on Robotics and Automation ICRA, New-Orleans, USA, 2004, pp. 504-510. 